\title{
Theory overview on FCNC B-decays
}

\author{
Admir Greljo*† \\ Physik-Institut, Universität Zürich, CH-8057 Zürich, Switzerland \\ E-mail: admirephysik.uzh.ch
}

LHCb experiment at CERN has recently reported a set of measurements on lepton flavour universality in $b \rightarrow s$ transitions showing a departure from the Standard Model predictions. I will review the main ideas recently put forward to make sense out of these intriguing hints. Focusing on the new physics explanation, I will discuss the correlated signals expected in other low- and high-energy observables, that could help clarify the mysterious anomalies.

The European Physical Society Conference on High Energy Physics

5-12 July

Venice, Italy

\footnotetext{
* Speaker.

${ }^{\dagger}$ I would like to thank Dario Buttazzo, Gino Isidori, and David Marzocca for the fruitful collaboration which led to several results presented in this talk. I would also like to thank conveners for the invitation.
} 


\section{Hints on NP in FCNC $B$-decays: Interpretations and implications}

Flavour changing neutral current (FCNC) $B$ meson decays are generated only at one-loop level in the Standard Model (SM) and suffer from additional GIM and parametric CKM suppression. Therefore, these processes are an excellent territory to search for new physics (NP) effects. Under the assumption of heavy new physics, IR dynamics at the $B$ mass scale is well described by the effective Hamiltonian approach in which the effects of NP are captured by a finite numbers of unknown coefficients. On the other hand, these can be efficiently extracted from the global fit to plethora of low energy measurements. For a recent review on the subject see Ref. [1].

Recent measurements in rare semileptonic $b \rightarrow s$ transitions provide strong hints for a new physics contribution to $b s \mu \mu$ local interactions. This is supported by the several global fits recently preformed in the literature (e.g. Refs. [2, 3, 4, 5]). In particular, a good fit to the differential observable $P_{5}^{\prime}$ [6], together with the hints on LFU violation in $R_{K}$ and $R_{K^{*}}$ [7, 8, 9], is obtained by considering a new physics contribution to the $C_{b s \mu} \equiv \mathbf{C}_{32}^{D \mu}$ coefficient in

$$
\mathscr{L}^{\text {eff }} \supset \frac{\mathbf{C}_{i j}^{U \mu}}{v^{2}}\left(\bar{u}_{L}^{i} \gamma_{\mu} u_{L}^{j}\right)\left(\bar{\mu}_{L} \gamma^{\mu} \mu_{L}\right)+\frac{\mathbf{C}_{i j}^{D \mu}}{v^{2}}\left(\bar{d}_{L}^{i} \gamma_{\mu} d_{L}^{j}\right)\left(\bar{\mu}_{L} \gamma^{\mu} \mu_{L}\right) .
$$

In terms of the SMEFT operators at the electroweak scale, this corresponds to a contribution to (at least) one of the two operators (see e.g. Ref. [10])

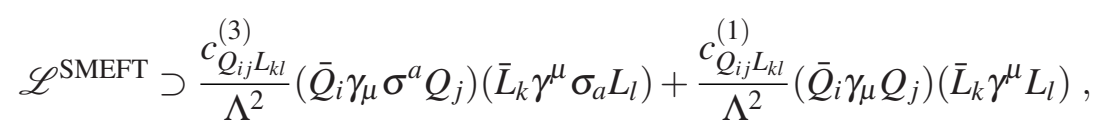

where $Q_{i}=\left(V_{j i}^{*} u_{L}^{j}, d_{L}^{i}\right)^{T}$ and $L_{i}=\left(v_{L}^{i}, \ell_{L}^{i}\right)^{T}$ are the SM left-handed quark and lepton weak doublets, respectively. Also, $V$ is the CKM flavour mixing matrix and $\sigma^{a}$ are the Pauli matrices acting on $S U(2)_{L}$ space. Moreover, it is worth noticing that the triplet operator could at the same time solve the anomalies in charged-currrent transitions $R_{D^{(*)}}$ (e.g. Refs. [11, 12, 13]). As discussed in the next section, this observation is the starting point to pursue the combined explanation of the two anomalies.

Matching at the tree level this operator to the standard effective weak Hamiltonian describing $b \rightarrow s$ transitions, one finds $\Delta C_{9}^{\mu}=-\Delta C_{10}^{\mu}=\frac{\pi}{\alpha V_{t b} V_{t s}^{*}} C_{b s \mu}$ where $\alpha$ is the electromagnetic fine structure constant while $\left|V_{t s}\right|=(40.0 \pm 2.7) \times 10^{-3}$ and $\left|V_{t b}\right|=1.009 \pm 0.031$ are CKM matrix elements. The recent combined fit of Ref. [2] reported the best fit value and $1 \sigma$ preferred range $\Delta C_{9}^{\mu}=-\Delta C_{10}^{\mu}=-0.61 \pm 0.12$. Using this result, one can estimate the scale of the relevant new physics by defining $C_{b s \mu}=g_{*}^{2} v^{2} / \Lambda^{2}$, obtaining $\Lambda / g_{*} \approx 32_{-3}^{+4} \mathrm{TeV}$. Depending on the value of $g_{*}$ (encoding the UV origin of the operator), the scale of new physics $\Lambda$ can be within or out of the reach of direct searches at the LHC.

Let us speculate in more details about the origin of these effective interactions. The full set of single mediator models with tree-level matching to the vector triplet $\left(c_{Q_{i j} L_{k l}}^{(3)}\right)$ or singlet $\left(c_{Q_{i j} L_{k l}}^{(1)}\right)$ operators, consists of: color-singlet vectors $B_{\mu}^{\prime} \sim(\mathbf{1}, \mathbf{1}, 0)$ and $W_{\mu}^{\prime} \sim(\mathbf{1}, \mathbf{3}, 0)$, color-triplet scalar $S_{3} \sim(\overline{\mathbf{3}}, \mathbf{3}, 1 / 3)$, and vectors $U_{1}^{\mu} \sim(\mathbf{3}, \mathbf{1}, 2 / 3), U_{3}^{\mu} \sim(\mathbf{3}, \mathbf{3}, 2 / 3)$, in the notation of Ref. [14]. The quantum numbers in brackets indicate color, weak, and hypercharge representations, respectively. It is important to notice the correlation in triplet versus singlet operators induced by a single mediator model as illustrated in Figure 2 (right). 


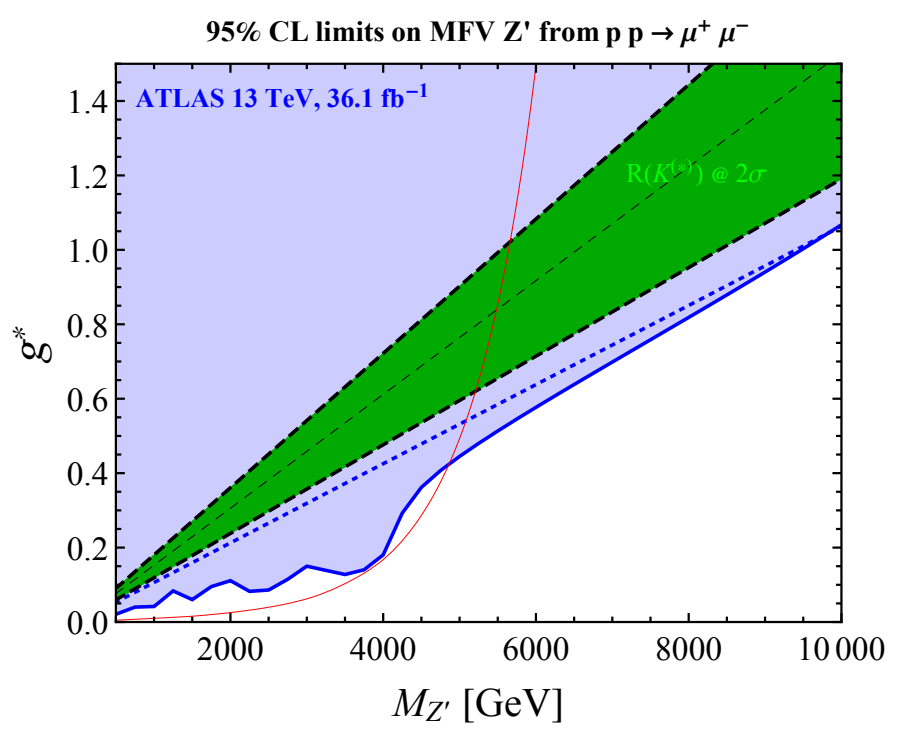

Figure 1: Limits on the $Z^{\prime}$ MFV model from $p p \rightarrow \mu^{+} \mu^{-}$. The plot is taken from Ref. [38].

Even if the mass scale of these mediators is beyond the kinematical reach of the LHC direct production, a signal at high- $\mathrm{p}_{T}$ might still be observed. In this context, impact of the contact interactions (due to the heavy scale) on the tails of dilepton invariant mass distribution in $p p \rightarrow$ $\ell^{+} \ell^{-}$has been studied in Ref. [38]. The limits obtained in this way are used to derive bounds on class of models which aim to solve the $b \rightarrow$ sll anomalies. ${ }^{1}$

A color-singlet vector resonance $Z^{\prime}$ gives rise to an $s$-channel resonant contribution to the dilepton invariant mass distributions if $M_{Z^{\prime}}$ is kinematically accessible. On the contrary, the deviation in the tails is described by the dimension-six operators in Eq. (1.2) with $\Lambda=M_{V}$, and $c_{Q_{i j} L_{k l}}^{(1)}=-g_{Q}^{(1), i j} g_{L}^{(1), k l}$. These are obtained after integrating out the heavy vector with interaction $\mathscr{L} \supset Z_{\mu}^{\prime} J_{\mu}$, where

$$
J_{\mu}=g_{Q}^{(1), i j}\left(\bar{Q}_{i} \gamma_{\mu} Q_{j}\right)+g_{L}^{(1), k l}\left(\bar{L}_{k} \gamma^{\mu} L_{l}\right) .
$$

A flavour-violating $g_{Q}^{(1), 23}$ coupling and $g_{L}^{(1), 22}$ are required to explain the flavour anomalies, while the limits from $p p \rightarrow \mu^{+} \mu^{-}$, can easily be translated to the flavour-diagonal coupling and mass combination. For example, assuming a $Z^{\prime}$ with $g_{Q}^{(1), i i}=g_{L}^{(1), i i}=g^{*}$ and MFV structure $\left(g_{Q}^{(1), 23}=\right.$ $\left.V_{t s} g^{*}\right)$, as dictated by neutral meson oscillation constraints, we derive limits on $g^{*}$ as a function of the mass $M_{Z^{\prime}}$, both fitting the data directly in the full model, ${ }^{2}$ and in the EFT approach. The results are shown in Figure 1. The limits in the full model are shown with solid-blue while those in the EFT are shown with dashed-blue. We see that for a mass $M_{Z^{\prime}} \gtrsim 4-5 \mathrm{TeV}$ the limits in the two approaches agree well, while for the lower masses the EFT still provides conservative bounds. On top of this, we show with green lines the best fit and $2 \sigma$ interval which reproduce the $b \rightarrow s \mu \mu$ flavour anomalies, showing how LHC dimuon searches already exclude such a scenario independently of

\footnotetext{
${ }^{1}$ With a similar spirit, in Ref. [15] it was shown that the LHC measurements of $p p \rightarrow \tau^{+} \tau^{-}$already set stringent constraints on models aimed at solving the charged-current $b \rightarrow c \tau \bar{v}_{\tau}$ anomalies.

${ }^{2}$ The $Z^{\prime}$ decay width is determined by decays into the SM fermions $u, d, s, c, b, t, \mu, v_{\mu}$ via Eq. (1.3), i.e. $\Gamma_{Z^{\prime}} / M_{Z^{\prime}}=$ $5 g_{*}^{2} /(6 \pi)$.
} 

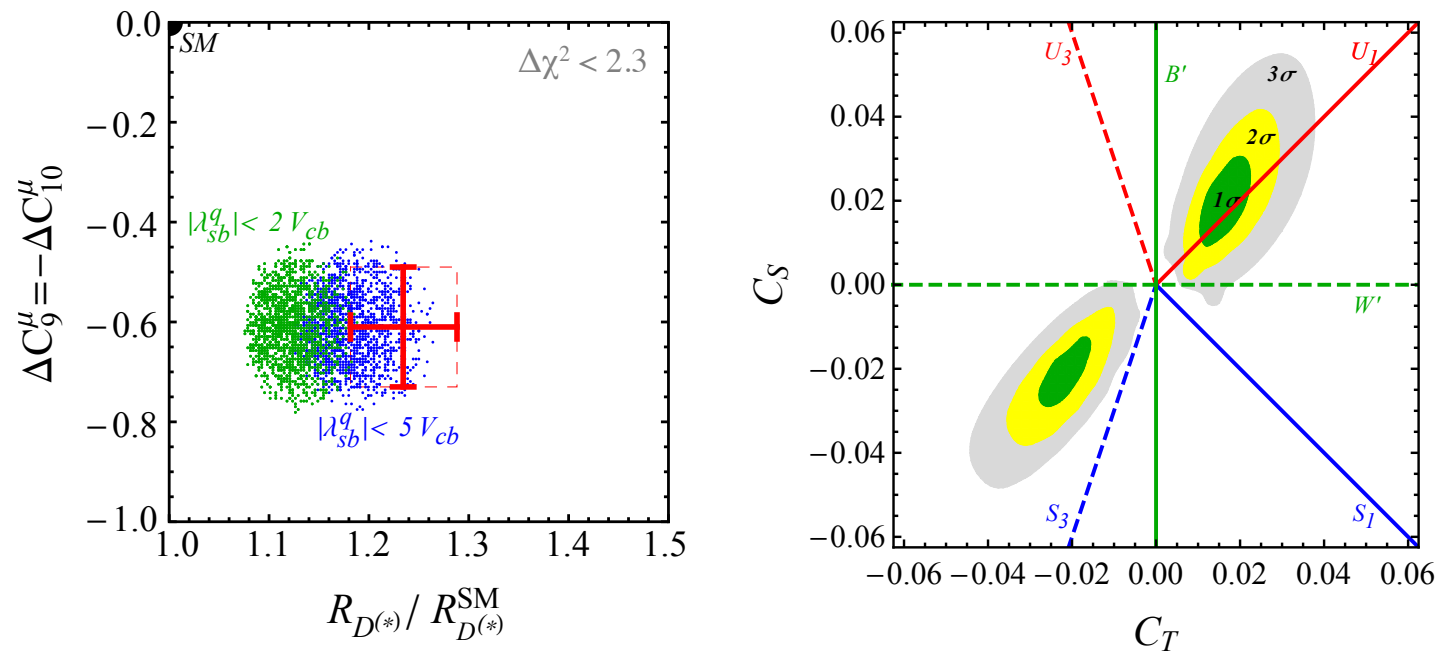

Figure 2: Left: Prediction for $\Delta C_{9}^{\mu}=-\Delta C_{10}^{\mu}$ (related to $b \rightarrow s \mu \mu$ data) and $R\left(D^{(*)}\right)$ for a randomly chosen set of points within the $1 \sigma$ preferred region of the EFT fit: the blue points are obtained setting $\left|\lambda_{s b}^{q}\right|<5\left|V_{c b}\right|$, while the green points are obtained setting the tighter condition $\left|\lambda_{s b}^{q}\right|<2\left|V_{c b}\right|$ in the fit. The red cross summarizes the present measurements. Right: Preferred region from the global fit projected in $\left(C_{T}, C_{S}\right)$ plane. The lines show the correlations among triplet and singlet operators in single-mediator models. Colourless vectors are shown in green, coloured scalar in blue, while coloured vectors in red. Electroweak singlet mediators are shown with the solid lines while triplets with dashed. The plots are taken from Ref. [17].

the $Z^{\prime}$ mass. The red solid line indicates the naive bound obtained when interpreting the limits on the narrow-width resonance production $\sigma\left(p p \rightarrow Z^{\prime}\right) \times \mathscr{B}\left(Z^{\prime} \rightarrow \mu^{+} \mu^{-}\right)$from Fig. 6 of Ref. [16].

\section{Combined explanation with $R\left(D^{(*)}\right)$ anomaly (hints in CC $B$-decays)}

There have been several attempts in the literature towards a combined explanation of $b \rightarrow c \ell \bar{v}$ and $b \rightarrow$ ste anomalies (see e.g. [17, 11, 12, 13, 18, 19, 20, 21, 22, 23, 24, 25, 26, 27, 28, 29, 30, 31, 32, 33]). Since the implied scale of new physics is rather low [34], the main challenge is to reconcile it with the non-observation of related signals in (other) flavour changing processes (e.g. [35]), electroweak precision observables, $\tau$ decays [36, 37], and high- $p_{T}$ searches $[15,38]$.

A combined explanation of both charged- and neutral-current $B$-physics anomalies, consistent with the absence of deviations from the SM so far observed in other observables, is possible and does not require unnatural tunings. As discussed in Ref. [17], a coherent picture is emerging when invoking: (i) leading NP effects in semi-leptonic operators built from the left-handed quark and lepton doublets (see Eq. (1.2)) and (ii) dominant couplings to third generation SM fermions with subleading terms for the light generations controlled by a minimally broken $U(2)_{q} \times U(2)_{\ell}$ flavour symmetry.

As shown in Ref. [17], a global fit to all relevant low-energy observables (including radiatively generated terms) leads to a good fit to all available data, without tuned cancellations and in terms of a small number of free parameters (see Figure 2 left). The preferred EFT solution suggests: (i) a sizeable heavy-light mixing in the quark sector (large $\lambda_{b s}^{q}$ ) that, despite being consistent with 
the minimal breaking of the flavour symmetry, helps to increase the effective scale of NP and (ii) a flavour-mixing structure different from the assumption of complete alignment of NP along a well-defined direction in flavour space. Two unambiguous low-energy signatures of this EFT construction are: (i) a huge enhancement (of two orders of magnitude or more) of FCNC transitions of the type $b \rightarrow s \tau \bar{\tau}$, (ii) the quark-flavour universality of the LFU ratios in charged currents, $R_{b \rightarrow u}^{\tau \ell}=R_{b \rightarrow c}^{\tau \ell}$, independently of initial- and final-state hadrons.

This EFT solution to the anomalies can be realised in terms of different simplified models (see Figure 2 right). A remarkably simple explanation of all the low-energy data is obtained by supplementing the SM with a single field - vector leptoquark representation $U_{1}^{\mu}=(\mathbf{3}, \mathbf{1}, 2 / 3)$. Importantly, leptoquarks [14] induce semi-leptonic transitions at tree level, while pure 4-quark and 4-lepton transitions arise only at one loop. However, the exceptional feature of this particular representation is the absence of tree-level down-quark-to-neutrino, as well as up-quark-to-charged-lepton transitions, naturally suppressing (a set of) otherwise strongly constrained observables. Other than being minimal in both the number of mediators and of free parameters, this model automatically presents some of the features suggested by the more general EFT fit, such as the relation $C_{S}=C_{T}$ and the absence of a flavour-blind contraction among light fermions. The model can easily escape present and near-future LHC searches for $\tau \bar{\tau}$ [15] (and third generation leptoquarks) as a consequence of the larger new physics scale implied by the low-energy fit, solving at the same time the two most pressing problems pointed out recently in the literature [36, 15].

Simplified models with a pair of scalar leptoquarks in the singlet and triplet representations of the $S U(2)_{L}$ gauge group emerge as a natural UV alternative to recover the same low-energy EFT. We find that also this setup provides an overall good description of data, albeit with a larger number of free parameters. The main advantage of this model is that the loop contribution to $\Delta F=2$ processes is calculable and small.

A possible UV completion for these simplified models can be realised in the context of composite Higgs models based on vector-like confinement: the mediators of the flavour anomalies could arise as composite states of a new strongly coupled sector confining at the $\mathrm{TeV}$ scale, of which the Higgs doublet is one of the pseudo-Nambu-Goldstone bosons [31, 32, 33]. Another avenue for the UV completion of the vector leptoquark model could be an extended gauge sector models in which the leptoquark is a massive gauge boson [39].

\section{Conclusions}

Standard model might be cracking down in FCNC B-decays. A coherent picture is emerging based on new dynamics involving left-handed currents and $U(2)$ flavour symmetry pattern. Importantly, such picture has a clear predictions in other low- and high-energy observables which can be tested in the near-future data and thus help to understand the intriguing anomalies in $B$-physics.

While waiting for more data, it is exciting (though premature) to speculate about the "Who ordered that?" question. Is this about the SM flavour puzzle? On the other hand, NP at the TeV scale is probably related to the Higgs mass hierarchy problem. So what is the connection? 


\section{References}

[1] T. Blake, G. Lanfranchi and D. M. Straub, Prog. Part. Nucl. Phys. 92, 50 (2017) doi:10.1016/j.ppnp.2016.10.001 [arXiv:1606.00916 [hep-ph]].

[2] B. Capdevila, A. Crivellin, S. Descotes-Genon, J. Matias and J. Virto, arXiv:1704.05340 [hep-ph].

[3] W. Altmannshofer, P. Stangl and D. M. Straub, Phys. Rev. D 96, no. 5, 055008 (2017) doi:10.1103/PhysRevD.96.055008 [arXiv:1704.05435 [hep-ph]].

[4] L. S. Geng, B. Grinstein, S. Jäger, J. Martin Camalich, X. L. Ren and R. X. Shi, arXiv:1704.05446 [hep-ph].

[5] M. Ciuchini, A. M. Coutinho, M. Fedele, E. Franco, A. Paul, L. Silvestrini and M. Valli, arXiv: 1704.05447 [hep-ph].

[6] S. Descotes-Genon, J. Matias, M. Ramon and J. Virto, JHEP 1301, 048 (2013) doi:10.1007/JHEP01(2013)048 [arXiv:1207.2753 [hep-ph]].

[7] G. Hiller and F. Kruger, Phys. Rev. D 69, 074020 (2004) doi:10.1103/PhysRevD.69.074020 [hep-ph/0310219].

[8] C. Bobeth, G. Hiller and G. Piranishvili, JHEP 0712, 040 (2007) doi:10.1088/1126-6708/2007/12/040 [arXiv:0709.4174 [hep-ph]].

[9] M. Bordone, G. Isidori and A. Pattori, Eur. Phys. J. C 76, no. 8, 440 (2016) doi:10.1140/epjc/s10052-016-4274-7 [arXiv:1605.07633 [hep-ph]].

[10] A. Celis, J. Fuentes-Martin, A. Vicente and J. Virto, Phys. Rev. D 96, no. 3, 035026 (2017) doi:10.1103/PhysRevD.96.035026 [arXiv:1704.05672 [hep-ph]].

[11] B. Bhattacharya, A. Datta, D. London and S. Shivashankara, Phys. Lett. B 742, 370 (2015) doi:10.1016/j.physletb.2015.02.011 [arXiv:1412.7164 [hep-ph]].

[12] R. Alonso, B. Grinstein and J. Martin Camalich, JHEP 1510, 184 (2015) doi:10.1007/JHEP10(2015)184 [arXiv:1505.05164 [hep-ph]].

[13] A. Greljo, G. Isidori and D. Marzocca, JHEP 1507, 142 (2015) doi:10.1007/JHEP07(2015)142 [arXiv:1506.01705 [hep-ph]].

[14] I. Dorsner, S. Fajfer, A. Greljo, J. F. Kamenik and N. Kosnik, Phys. Rept. 641, 1 (2016) doi:10.1016/j.physrep.2016.06.001 [arXiv:1603.04993 [hep-ph]].

[15] D. A. Faroughy, A. Greljo and J. F. Kamenik, Phys. Lett. B 764, 126 (2017) doi:10.1016/j.physletb.2016.11.011 [arXiv:1609.07138 [hep-ph]].

[16] The ATLAS collaboration [ATLAS Collaboration], ATLAS-CONF-2017-027.

[17] D. Buttazzo, A. Greljo, G. Isidori and D. Marzocca, arXiv:1706.07808 [hep-ph].

[18] L. Calibbi, A. Crivellin and T. Ota, Phys. Rev. Lett. 115, 181801 (2015) doi:10.1103/PhysRevLett.115.181801 [arXiv:1506.02661 [hep-ph]].

[19] M. Bauer and M. Neubert, Phys. Rev. Lett. 116, no. 14, 141802 (2016) doi:10.1103/PhysRevLett.116.141802 [arXiv:1511.01900 [hep-ph]].

[20] S. Fajfer and N. Koĺnik, Phys. Lett. B 755, 270 (2016) doi:10.1016/j.physletb.2016.02.018 [arXiv:1511.06024 [hep-ph]]. 
[21] D. Das, C. Hati, G. Kumar and N. Mahajan, Phys. Rev. D 94, 055034 (2016) doi:10.1103/PhysRevD.94.055034 [arXiv:1605.06313 [hep-ph]].

[22] S. M. Boucenna, A. Celis, J. Fuentes-Martin, A. Vicente and J. Virto, JHEP 1612, 059 (2016) doi:10.1007/JHEP12(2016)059 [arXiv:1608.01349 [hep-ph]].

[23] D. Becirevic, S. Fajfer, N. Kosnik and O. Sumensari, Phys. Rev. D 94, no. 11, 115021 (2016) doi:10.1103/PhysRevD.94.115021 [arXiv:1608.08501 [hep-ph]].

[24] G. Hiller, D. Loose and K. Schönwald, JHEP 1612, 027 (2016) doi:10.1007/JHEP12(2016)027 [arXiv:1609.08895 [hep-ph]].

[25] B. Bhattacharya, A. Datta, J. P. Guévin, D. London and R. Watanabe, JHEP 1701, 015 (2017) doi:10.1007/JHEP01(2017)015 [arXiv:1609.09078 [hep-ph]].

[26] A. Crivellin, D. Muller and T. Ota, JHEP 1709, 040 (2017) doi:10.1007/JHEP09(2017)040 [arXiv:1703.09226 [hep-ph]].

[27] D. Becirevic, N. Kosnik, O. Sumensari and R. Zukanovich Funchal, JHEP 1611, 035 (2016) doi:10.1007/JHEP11(2016)035 [arXiv:1608.07583 [hep-ph]].

[28] Y. Cai, J. Gargalionis, M. A. Schmidt and R. R. Volkas, arXiv:1704.05849 [hep-ph].

[29] D. Aloni, A. Dery, C. Frugiuele and Y. Nir, arXiv:1708.06161 [hep-ph].

[30] I. Dorsner, S. Fajfer, D. A. Faroughy and N. Kolínik, arXiv:1706.07779 [hep-ph].

[31] R. Barbieri, G. Isidori, A. Pattori and F. Senia, Eur. Phys. J. C 76, no. 2, 67 (2016) doi:10.1140/epjc/s10052-016-3905-3 [arXiv:1512.01560 [hep-ph]].

[32] D. Buttazzo, A. Greljo, G. Isidori and D. Marzocca, JHEP 1608, 035 (2016) doi:10.1007/JHEP08(2016)035 [arXiv:1604.03940 [hep-ph]].

[33] R. Barbieri, C. W. Murphy and F. Senia, Eur. Phys. J. C 77, no. 1, 8 (2017) doi:10.1140/epjc/s10052-016-4578-7 [arXiv:1611.04930 [hep-ph]].

[34] L. Di Luzio and M. Nardecchia, Eur. Phys. J. C 77, no. 8, 536 (2017) doi:10.1140/epjc/s10052-017-5118-9 [arXiv:1706.01868 [hep-ph]].

[35] R. Alonso, B. Grinstein and J. Martin Camalich, Phys. Rev. Lett. 118, no. 8, 081802 (2017) doi:10.1103/PhysRevLett.118.081802 [arXiv:1611.06676 [hep-ph]].

[36] F. Feruglio, P. Paradisi and A. Pattori, Phys. Rev. Lett. 118, no. 1, 011801 (2017) doi:10.1103/PhysRevLett.118.011801 [arXiv:1606.00524 [hep-ph]].

[37] F. Feruglio, P. Paradisi and A. Pattori, JHEP 1709, 061 (2017) doi:10.1007/JHEP09(2017)061 [arXiv:1705.00929 [hep-ph]].

[38] A. Greljo and D. Marzocca, Eur. Phys. J. C 77, no. 8, 548 (2017) doi:10.1140/epjc/s10052-017-5119-8 [arXiv:1704.09015 [hep-ph]].

[39] L. Di Luzio, A. Greljo and M. Nardecchia, arXiv:1708.08450 [hep-ph]. 